\section{Guillaine-Barre Syndrome with Retained Deep Tendon Reflexes}

Guillain-Barré syndrome (GBS) is an acute onset, usually monophasic immune-mediated disorder of the peripheral nervous system. Acute inflammatory demyelinating polyradiculoneuropathy (AIDP) is the most widely known form of GBS. However, axonal variants of GBS are also commonly seen in India; in one Indian series, the incidence of AIDP and AMAN (Acute motor axonal neuropathy) was virtually equal [1].

The widely employed Asbury and Cornblath case definitions and the recent, the Brighton Collaboration developed case definitions of GBS were developed in the West, where AIDP is the most common form of GBS $[2,3]$. The two features essential for a diagnosis of GBS according to these criteria are progressive motor weakness and areflexia. The axonal variant of GBS usually has retained deep tendon reflexes or at times hyperreflexia either in the beginning or throughout the course of the disease [4]. Though this was described in the initial studies of AMAN, most text books still stress the presence of areflexia in GBS. This results in delay in the diagnosis of GBS or may even result in considering upper motor neuron disease as a cause for the presentation resulting in unnecessary investigations like magnetic resonance imaging of the spine/ brain resulting in additional cost to the parents.

Recently, there have been reports of hyperreflexia in GBS - mainly in the adult literature - from India also but not in children $[5,6]$. The variants most commonly reported to be associated with retained or brisk reflexes are AMAN, acute motor conduction block neuropathy, and acute facial diplegia with brisk reflexes, of which AMAN is of more importance to pediatricians. Although preservation of reflexes, may simply be due to sparing of the sensory afferent pathway, the occurrence of brisk reflexes suggests a central mechanism. A proposed mechanism for hyperreflexia is dysfunction of inhibitory systems in the spinal interneurons. Distal conduction disturbance - not axonal degeneration-produces low motor responses on nerve conduction studies, termed as reversible conduction failure or acute motor conduction block neuropathy [6]. This could explain the relatively good prognosis in this group.

Thus even though hyporeflexia or areflexia is necessary for diagnosis of typical Guillain Barre Syndrome, hyperreflexia does not exclude a GBS variant and usually suggests an axonal type of GBS.

\author{
Mahesh Kamate \\ Department of Pediatrics, \\ KLE University's $J$ Medical College, \\ Belgaum, Karnataka, India. \\ drmaheshkamate@gmail.com
}

\section{REFERENCES}

1. Sinha S, Prasad KN, Jain D, Pandey CM, Jha S, Pradhan S. Preceding infections and gangliosides antibodies in patients with Guillain-Barré syndrome: A single center prospective case-control study. Clin Microbial Infect. 2007;13:334-7.

2. Asbury AK, Cornblath DR. Assessment of current diagnostic criteria for Guillain-Barre syndrome. Ann Neurol. 1990;27:S21-4.

3. Sejvar JJ, Kohl KS, Gidudu J, Amato A, Bakshi N, Baxter $\mathrm{R}$, et al. Guillain-Barre syndrome and Fisher syndrome: Case definitions and guidelines for collection, analysis, and presentation of immunization safety data. Vaccine. 2011;29:599-612.

4. Kuwabara S, Nakata M, Sung JY, Mori M, Kato $\mathrm{N}$, Hattori T, et al. Hyperreflexia in axonal Guillain-Barré syndrome subsequent to Campylobacter jejuni enteritis. J Neurol Sci. 2002;199:89-92.

5. Somarajan A. Guillain Barre syndrome with brisk reflexesanother variant. Neurol India. 2006;54:215-6.

6. Baheti NN, Manual D, Shinde PD, Radhakrishnan A, Nair M. Hyperreflexic Guillain-Barré syndrome. Ann Indian Acad Neurol. 2010;13:305-7. 